\title{
Singular Continuous Spectrum for Palindromic Schrödinger Operators
}

\author{
A. Hof ${ }^{1}$, O. Knill ${ }^{2}$, B. Simon ${ }^{2}$ \\ ${ }^{1}$ Department of Mathematics and Statistics, McMaster University, Hamilton, Ontario, Canada L8S \\ $4 \mathrm{~K} 1$. Work partially supported by NSERC. \\ 2 Division of Physics, Mathematics and Astronomy, California Institute of Technology, 253-37, \\ Pasadena, CA, 91125, USA. This material is based upon work supported by the National Science \\ Foundation under Grant No. DMS-9101715. The Government has certain rights in this material.
}

Received: 28 July 1994/in revised form: 28 November 1994

\begin{abstract}
We give new examples of discrete Schrödinger operators with potentials taking finitely many values that have purely singular continuous spectrum. If the hull $X$ of the potential is strictly ergodic, then the existence of just one potential $x$ in $X$ for which the operator has no eigenvalues implies that there is a generic set in $X$ for which the operator has purely singular continuous spectrum. A sufficient condition for the existence of such an $x$ is that there is a $z \in X$ that contains arbitrarily long palindromes. Thus we can define a large class of primitive substitutions for which the operators are purely singularly continuous for a generic subset in $X$. The class includes well-known substitutions like Fibonacci, Thue-Morse, Period Doubling, binary non-Pisot and ternary non-Pisot. We also show that the operator has no absolutely continuous spectrum for all $x \in X$ if $X$ derives from a primitive substitution. For potentials defined by circle maps, $x_{n}=1_{J}\left(\theta_{0}+n \alpha\right)$, we show that the operator has purely singular continuous spectrum for a generic subset in $X$ for all irrational $\alpha$ and every half-open interval $J$.
\end{abstract}

\section{Introduction}

Discrete Schrödinger operators with potentials taking values in a finite set $A \subset$ $\mathbb{R}$ have interesting spectral properties. Topological spaces of such operators are obtained by choosing a compact shift-invariant subset $X$ of the compact metric space $A^{\mathbb{Z}}$. If $T$ denotes the left shift on $X$, the dynamical system $(X, T)$ is called a subshift. Every point $x \in X$ defines an operator on $l^{2}(\mathbb{Z})$ by

$$
(L(x) u)_{n}=u_{n+1}+u_{n-1}+x_{n} u_{n} .
$$

In $X=A^{\mathbb{Z}}$, any spectral type can occur: the dense set of periodic operators in $A^{\mathbb{Z}}$ have purely absolutely continuous spectrum; for almost all $x$ with respect to any non-trivial product measure on $X=A^{\mathbb{Z}}$ the spectrum is pure point [7]. Hence, by the wonderland theorem [24], there exists a generic set in $A^{\mathbb{Z}}$ for which $L(x)$ has purely singular continuous spectrum. The shift on $A^{\mathbb{Z}}$ has many invariant measures and there are many orbits which are not dense. It is therefore convenient to consider the case of a compact shift-invariant $X \subset A^{\mathbb{Z}}$ that is minimal (i.e., the set of 
translates of every $x \in X$ is dense in $X$ ) and uniquely ergodic (i.e., there exists only one $T$-invariant measure). A system $(X, T)$ that is both minimal and uniquely ergodic is called strictly ergodic. Two common methods for generating strictly ergodic subshifts are the following:

- A primitive substitution, which is a map $S$ from the alphabet $A$ to the set of finite words $A^{*}$, defines a fixed point $z^{+} \in A^{\mathbb{N}}$. Taking any $z \in A^{\mathbb{Z}}$ satisfying $z_{n}=z_{n}^{+}$ for $n \in \mathbb{N}$ and defining $X_{S}$ as the set of accumulation points of $\left\{T^{n} z \mid n \in \mathbb{N}\right\}$ gives a strictly ergodic system.

- A circle map $\theta \mapsto \theta+\alpha$ with irrational $\alpha$ together with a countable union $J$ of half-open intervals and an initial point $\theta_{0} \in \mathbb{T}^{1}$ defines a sequence $z_{n}=1_{J}\left(\theta_{0}+\right.$ $n \alpha)$. The orbit closure $X_{J}=X\left(J, \theta_{0}, \alpha\right)$ is independent of $\theta_{0}$ and is a strictly ergodic dynamical system (see [15] Proposition A.1.)

The aim of this paper is to give new examples of discrete Schrödinger operators that have purely singular continuous spectrum. We therefore need to exclude absolutely continuous spectrum and eigenvalues.

Absolutely continuous spectrum is excluded by Kotani's theorem [19] which says that almost all $L(x)$ have no absolutely continuous spectrum, since the potential takes finitely many values. Although the spectrum of $L(x)$ does not depend on $x \in X$ if $X$ is strictly ergodic [15], it does not follow that there is no absolutely continuous spectrum for all $x \in X$. If, however, the Lyapunov exponent exists for all $x \in X$ and is independent of $x$, then indeed no $L(x)$ has absolutely continuous spectrum as we will see below. In [15] it is shown that the Lyapunov exponent exists uniformly if $X$ is defined by a primitive substitution. Thus we exclude absolutely continuous spectrum for all operators $L(x)$ generated by primitive substitutions. We want to emphasize that it is an open problem (cf. Remark 2 in Sect. 6) whether for every strictly ergodic potential the absolutely continuous spectrum is independent of $x \in X$.

In order to get singular continuous spectrum one has also to exclude eigenvalues. This was done by a Gordon-type criterion for circle maps [10] and by using the so-called trace map in $[8,26,27,4,1,11,2,5]$ for circle maps and substitutions. In this paper we note that a variation on the criterion of Jitomirskaya and Simon [17] can be used for sequences that we call strongly palindromic. A strongly palindromic sequence contains palindromes $w_{l}$ of length $l_{i}$ centered at $m_{i} \rightarrow \infty$ such that $l_{i}$ grows exponentially fast with respect to $m_{l}$. We show that if a sequence $x \in X$ contains arbitrary large palindromes (we call this palindromic), then there is an uncountable set in $X$ which is strongly palindromic. Many kinds of sequences defined by circle maps and substitutions are palindromic. In addition, we show that the existence of just one $x \in X$ for which $L(x)$ has no eigenvalues implies that there is a generic set in $X$ for which $L(x)$ has purely singular continuous spectrum.

Our work gives new examples of operators with purely singular continuous spectrum for both substitutions and circle maps.

For circle maps, Delyon and Petritis [10] have shown absence of eigenvalues for almost all $\alpha$, all intervals $[0, \beta)$ and almost every $\theta_{0}$. They do not exclude eigenvalues for all irrational $\alpha$. Eigenvalues have been excluded for all irrational $\alpha$ in the case $\beta=\alpha$ by Bellissard et al. [4], but only for $\theta_{0}=0$. This was proved independently by Süto" [27] in the "golden case" $\alpha=(\sqrt{5}-1) / 2$. We prove here that for all irrational $\alpha$ and all intervals $J=[0, \beta)$, there is a generic set in $X_{J}$ for which $L(x)$ has purely singular continuous spectrum.

All papers on Schrödinger operators with potentials defined by primitive substitutions $[1,2,11,5]$ exclude eigenvalues for just one $x ;[2,5]$ claim that the spectrum 
is a Cantor set of Lebesgue measure 0 . We get purely singular continuous spectrum for a generic set in $X_{S}$ for a large class of substitutions. Only Bovier and Ghez [5] claim to exclude eigenvalues for a class of substitutions. Our class of substitutions contains all but one of the examples (the "circle sequence") given in [5] and the Bovier-Ghez class does not contain the "period doubling" and the "Binary-NonPisot" substitutions, which are contained in ours. (It should be noted, however, that the results in $[2,5]$ do not apply to the sequences stated in those papers, but to different ones (see the erratum [6]) that are not necessarily in $X_{S}$.)

The paper is organized as follows. Section 2 introduces palindromic and strongly palindromic sequences. Examples of (strongly) palindromic sequences are given in Sect. 3 (substitution sequences) and 4 (sequences defined by circle maps). Section 5 shows that $L(x)$ has no eigenvalues if $x$ is strongly palindromic. In Sect. 6 we prove that $L(x)$ has no absolutely continuous spectrum for all $x \in X$ if $X$ is defined by a primitive substitution. Section 7 combines these results to show that $L(x)$ has purely singular continuous spectrum for a generic set of $x \in X$ for strictly ergodic subshifts $X$ defined by a large class of circle subshifts and primitive substitutions.

\section{Palindromic Sequences}

Let $A$ be a finite set called alphabet and $A^{*}$ the set of (finite) words $w_{1} w_{2} \cdots w_{n}$ with $w_{i} \in A$. A word $w \in A^{*}$ is called a palindrome if it is the same when read backwards. The empty word is considered a palindrome. An element $x \in A^{\mathbb{Z}}$ is called palindromic if $x$ contains arbitrarily long palindromes. We say that a word $w$ is centered at $(n+m) / 2$ in $x$ if $w=x_{n} \cdots x_{m}$.

The shift $T$ on $A^{\mathbb{Z}}$ is defined by $T(z)_{n}=z_{n+1}$. The orbit $\operatorname{Orb}(z)$ of $z \in A^{\mathbb{Z}}$ is the set $\left\{T^{n} z\right\}_{n \in \mathbb{Z}}$. The closure of $\operatorname{Orb}(z)$ in the product topology is called the orbit closure of $z$ and is denoted by $X_{z}$. A compact shift-invariant subset $X$ of $A^{\mathbb{Z}}$ is called minimal if $X_{z}=X$ for all $z \in X$.

If $X$ is minimal then every word occurring in some $y \in X$ occurs in all $x \in X$. Thus it makes sense to speak of palindromic sets $X \subset A^{\mathbb{Z}}$, compact shift-invariant minimal sets containing one (and therefore only) palindromic sequences. Similarly, $X$ is called aperiodic if the elements in $X$ are aperiodic. Let $B>0$ be arbitrary (its value will be fixed in the proof of Theorem 5.1). Define $x \in A^{\mathbb{Z}}$ to be strongly palindromic, if there exists a sequence $w_{l}$ of palindromes of length $l_{l}$ centered at $m_{l} \rightarrow \infty$ such that $e^{B m_{l}} / l_{l} \rightarrow 0$. If $x$ is strongly palindromic then it is clearly palindromic.

If $X$ is minimal, then for every word $w$ there is a $d(w) \in \mathbb{N}$ such that in every $x \in X$ there occurs a copy of $w$ in every interval of length $d(w)$ (see, e.g., [23], p. 71). This fact is used in Proposition 2.1 to construct uncountably many different strongly palindromic sequences in a palindromic minimal subshift. The idea is the following. Suppose $w$ is a palindrome centered at $m$. Any long palindrome $w^{\prime}$ (say of length $l^{\prime}$ ) will contain two copies of $w$ within distance $2 d(w)$ to the left of its center. So $w$ can be extended to $w^{\prime}$ in two ways such that the center $m^{\prime}$ of $w^{\prime}$ is bounded by $2 d(w)$. Hence $l^{\prime}$ can be chosen to make $e^{B m^{\prime}} / l^{\prime}$ as small as desired. This process can be repeated to construct strongly palindromic sequences.

Proposition 2.1. If an aperiodic minimal set $X \subset A^{\mathbb{Z}}$ is palindromic, then it contains uncountably many strongly palindromic sequences. 
Proof. Let $\left\{w^{i}\right\}_{l=1}^{\infty}$ be a sequence of palindromes of length $l_{l} \rightarrow \infty$ that occur in the sequences in $X$. We will construct sequences $y_{n} \in A^{\mathbb{Z}}$ such that

1) $y_{n}$ is equal to one of the $w^{l}$ on an interval $I_{n}$ containing 0 ;

2) $I_{n} \subset I_{n+1}$ and every $m \in \mathbb{Z}$ is eventually in these intervals;

3) $y_{n+1}$ is equal to $y_{n}$ on $I_{n}$;

4) for every $y_{n}$, there are two possibilities for $y_{n+1}$ that are different on the intersection of the intervals where they are equal to one of the $w^{l}$.

Clearly, every such sequence $y_{n}$ converges to a different $y \in X$. Since there are infinitely many choices to be made, there are uncountably many different limit points. Conditions will be imposed to make sure that the limit points are strongly palindromic.

Choose a sequence of integers $m_{i}$ such that $m_{i} \rightarrow \infty$ and $e^{B m_{l}} / l_{i} \rightarrow 0$ as $i \rightarrow \infty$. Let $y_{n}$ be equal to $w^{j_{n}}$ on an interval $I_{n}$ of length $l_{j_{n}}$ containing 0 . We show how two choices arise for $y_{n+1}$. There exists an integer $i_{n+1}$ such that $d\left(w^{j_{n}}\right)<m_{l_{n+1}} / 2$. This means there is a copy of $w^{j n}$ in each of the first two subwords of length $m_{l_{n+1}} / 2$ to the left of the center of each $w^{j}$ with $j \geqq i_{n+1}$. There exists a $j_{n+1} \geqq i_{n+1}$ such that $w^{j_{n+1}}$ is not periodic with any period less than or equal to $m_{i_{n+1}}$ over an interval of length $l_{j_{n+1}}-m_{i_{n+1}}$, for otherwise $X$ would contain a periodic sequence by minimality. The two occurrences of $w^{j_{n}}$ in those first two subwords to the left of the center of $w^{j_{n+1}}$ give the two possibilities for $y_{n+1}$. Denote the two possibilities by $z$ and $z^{\prime}$. Let $I$ and $I^{\prime}$ denote the intervals on which $z$ and $z^{\prime}$ are equal to $w^{\prime n+1}$, respectively.

We are left to show that $z$ and $z^{\prime}$ differ on $I \cap I^{\prime}$. Note that the length of $I \cap I^{\prime}$ is at least $l_{j_{n+1}}-m_{i_{n+1}}$ and that $z^{\prime}$ on $I \cap I^{\prime}$ is equal to $z$ translated by at most $m_{i_{n+1}}$. Now suppose $z$ and $z^{\prime}$ are equal on $I \cap I^{\prime}$. Then $w^{j_{n+1}}$ would be periodic with period at most $m_{i_{n+1}}$ over a length of at least $l_{j_{n+1}}-m_{l_{n+1}}$. But $j_{n+1}$ was chosen such that this does not happen.

Note that each limit point of the $y_{n}$ must be strongly palindromic because $l_{I_{n}} \geqq l_{i_{n}}$ and because the center of $w^{j_{n}}$ is within distance $m_{j_{n}}$ of 0 .

\section{Substitution Sequences}

A substitution is a map $S: A \mapsto A^{*}$; it will be extended to a map $A^{*} \mapsto A^{*}$ and $A^{\mathbb{N}} \mapsto A^{\mathbb{N}}$ by concatenation, that is, $S\left(a_{1} a_{2} \cdots a_{n}\right)=S\left(a_{1}\right) S\left(a_{2}\right) \cdots S\left(a_{n}\right)$. A substitution $S$ is called primitive if there exists a $k$ such that for all $a \in A$ the word $S^{k}(a)$ contains at least one copy of every symbol. Without loss of generality, one can assume that there exists $a \in A$ such that $S(a)$ starts with $a$ (see [15]). Iterating $S$ on $a$ then gives a fixed point $z^{+} \in A^{\mathbb{N}}$. Let $z$ be any element in $A^{\mathbb{Z}}$ satisfying $z_{n}=z_{n}^{+}$for $n \in \mathbb{N}$. The substitution dynamical system $\left(X_{S}, T\right)$ is now defined by

$$
X_{S}=\left\{x \in A^{\mathbb{Z}} \mid x=\lim _{j \rightarrow \infty} T^{n_{j}} z \text { and } n_{j} \rightarrow \infty\right\}
$$

It is possible to generate the sequences in $X_{S}$ directly from the substitution (see, e.g., $[9,14])$. The system $\left(X_{S}, T\right)$ is strictly ergodic (see, e.g., [23]). A substitution $S$ is called palindromic if $X_{S}$ is palindromic.

We say that a primitive substitution $S$ is in the class $P$ if there exists a palindrome $p$ and for each $b \in A$ a palindrome $q_{b}$ such that $S(b)=p q_{b}$ for all $b \in A$. 
We allow $p$ to be the empty word; if $p$ is not the empty word then $q_{b}$ is allowed to be the empty word.

\section{Lemma 3.1. Class $P$ substitutions are palindromic.}

Proof. We show first by induction over the length of the word $w$ that if $w$ is a palindrome, then $S(w)=p u$ with a palindrome $u$. We can write $w=b v b$ with some $b \in A$ and some palindrome $v$. We have $S(w)=S(b) S(v) S(b)=p q_{b} S(v) p q_{b}$. By induction, $S(v)$ is of the form $p u$ with some palindrome $u$. We get $S(w)=$ $p q_{b} p u p q_{b}$ which is of the form $p \tilde{u}$ with palindrome $\tilde{u}$. All words $S^{k}(a)$ occur in all $x \in X_{S}$, so the sequences contain arbitrarily long palindromes.

Corollary 3.2. If $S$ is class $P$, then there are uncountably many elements in $X_{S}$ that are strongly palindromic.

Proof. Apply 2.1 and 3.1 .

Remarks. 1) It is sufficient that some power of $S$ is of class $P$ in order that $S$ is palindromic.

2) A subclass of class $P$ are substitutions for which $S(b)$ is a palindrome for all $b \in A$. Just take for $p$ the empty palindrome.

3 ) Clearly, we could include into class $P$ substitutions of the form $S(b)=q_{b} p$. We do not know whether all palindromic $X_{S}$ arise from substitutions that are in this extended class $P$.

4) Not all substitutions are palindromic, as the following example shows:

$$
S: a \mapsto a b b a a a b b b a, b \mapsto a b
$$

The word bbaaabbb occurs in the sequences but the word bbbaaabb does not occur.

5 ) The set of strongly palindromic sequences is invariant and therefore has either measure 0 or 1 ; but which we do not know. We cannot even exclude that all $x$ are strongly palindromic.

Here are some examples of substitutions in class $P$ that have appeared in the literature of Schrödinger operators:

Thue-Morse: $S^{2}: a \mapsto a b b a, b \mapsto b a a b[20,1,11]$.

Fibonacci: $S: a \mapsto a b, b \mapsto a[18,22,8,26,27,4,3,5]$.

Period doubling: $S: a \mapsto a b, b \mapsto a a[20,2,3,5]$.

Binary non-Pisot: $S: a \mapsto a b, b \mapsto a a a[3,5]$.

Ternary non-Pisot: $S: a \mapsto c, b \mapsto a, c \mapsto b a b[3,5]$.

Remarks. 6) The papers $[2,3,5]$ implicitly consider the set $X_{z}$, where $z$ is the symmetric extension of $z^{+}$: for $n \in \mathbb{N}, z_{-n-1}=z_{n}^{+}$and $z_{n}=z_{n}^{+}$. This is not natural. The system $\left(X_{z}, T\right)$ is, in general, neither uniquely ergodic nor minimal.

7) We do not know if the Rudin-Shapiro sequence $\left(r_{n}=(-1)^{f_{n}}\right.$, where $f_{n}$ is the number of pairs 11 in the binary expansion of $n$ ) is palindromic. Contrary to a statement in a preliminary version of this paper, our computer experiments suggest that it is not palindromic. A Schrödinger operator with potential based on the Rudin-Shapiro sequence has appeared in [20]. Numerical experiments done in $[12,13]$ indicate that there might be eigenvalues. 


\section{Sequences Defined by Circle Maps}

Consider the irrational rotation $\theta \mapsto \theta+\alpha$ on the circle $\mathbb{T}^{1}=\mathbb{R} / \mathbb{Z}$. For a half-open interval $J=[0, \beta) \subset \mathbb{T}^{1}$ and a point $\theta_{0}$ in $\mathbb{T}^{1}$, we consider the sequence $z=z\left(\theta_{0}\right)$ given by

$$
z_{n}=1_{J}\left(n \alpha+\theta_{0}\right) \in\{0,1\}^{\mathbb{Z}} .
$$

We denote by $X_{J}$ the compact subset of $A^{\mathbb{Z}}$ obtained by taking the closure of the orbit of $z\left(\theta_{0}\right)$. It is independent of $\theta_{0}$ and the system $\left(X_{J}, T\right)$ is strictly ergodic. This remains true if the half-open interval $J$ is replaced by a countable union of half-open intervals [15].

Lemma 4.1. For all irrational $\alpha$ and every half-open interval $J$ there exists $\theta_{0} \in \mathbb{T}^{1}$ such that $z\left(\theta_{0}\right)$ is palindromic.

Proof. If $\beta \neq 2 k \alpha$, then the orbit of $\theta_{0}=\beta / 2$ is disjoint from $\{0, \beta\}$. If $\beta=2 k \alpha$ $(\bmod 1)$, the orbit of $\theta_{0}=(\beta+1) / 2$ is disjoint from $\{0, \beta\}$. By symmetry, these orbits are even and therefore palindromic if they are disjoint from $\{0, \beta\}$.

Remarks. 1) The lemma can be generalized. Suppose $J$ is a symmetric countable union of half-open intervals with a point of symmetry $\theta_{0}$. Then if one of the orbits of the points $\theta_{0}+(i+j \alpha) / 2(i, j \in\{0,1\})$ does not hit the boundary of $J$, then that orbit is even, and $X_{J}$ is palindromic.

2) Another generalization of the lemma can be obtained by taking an ergodic group translation $\theta \mapsto \theta+\alpha$ on $\mathbb{T}^{v}$ and taking $J=\left[0, \beta_{1}\right) \times \cdots \times\left[0, \beta_{v}\right)$. The sequence $z_{n}=1_{J}(\theta+n \alpha)$ generates a strictly ergodic set $X$ independent of $\theta$ as in the case $v=1$. This set $X$ is palindromic: it can be shown (by induction in $v$ ) that one of the orbits starting at $\theta_{0}=(\beta+v) / 2$, where $v_{i} \in\{0,1\}$, does not hit the boundary of $J$.

3) There are (in cardinality) more sequences generated by circle maps than sequences generated by substitutions: the length of the interval $[0, \beta)$ measures the average frequency of occurrences of 1 in the sequence $z_{n}$. In this way one obtains uncountably many different sequences for different $\beta$. On the other hand, there are only countably many substitutions.

4) The circle $\mathbb{T}^{1}$ is embedded in $X_{J}$ by $z(\theta)=1_{J}(n \alpha+\theta)$ but not every sequence $z \in X_{J}$ is of this form.

\section{The Point Spectrum}

In the preceding three sections $A$ was a finite set of symbols. In the remainder of the paper we map the elements to $A$ to real numbers and again denote the image by $A$. So from now on sequences $x \in A^{\mathbb{Z}}$ take values in $\mathbb{R}$. Every $x \in A^{\mathbb{Z}}$ defines a discrete Schrödinger operator on $l^{2}(\mathbb{Z})$ by

$$
(L(x) u)_{n}=u_{n+1}+u_{n-1}+x_{n} u_{n} .
$$

Theorem 5.1 (Jitomirskaya-Simon). If $x$ is strongly palindromic, then L(x) has no eigenvalues.

Proof. The proof is essentially [17]. However, [17] deals with operators on $l^{2}(\mathbb{R})$ with uniformly almost periodic potentials. Since we consider discrete operators with potentials taking finitely many values, the proof in [17] needs some modifications. For the convenience of the reader, we have included a proof in the appendix. 
Remarks. 1) Most (with respect to the product measure) sequences in $A^{\mathbb{Z}}$ are palindromic since almost all sequences contain all possible finite words. But most sequences in $A^{\mathbb{Z}}$ are not strongly palindromic since most sequences give Schrödinger operators with Anderson localization [7] and strongly palindromic sequences give operators without eigenvalues.

2 ) In the golden case treated in $[27,4]$ the sequence $x$ is symmetric around 0. The sequence in [11] is antisymmetric around 0 . We do not know whether these sequences are strongly palindromic, so we can not exclude eigenvalues for those particular sequences, although in both cases $X$ is palindromic.

\section{The Absolutely Continuous Spectrum}

Theorem 6.1. If the dynamical system $(X, T)$ has the property that the Lyapunov exponent $\lambda_{x}(E)=\lambda(E)$ of $L(x)$ exists for all $x \in X$ and is independent of $x \in X$, then $L(x)$ has no absolutely continuous spectrum for all $x \in X$.

Proof. For all $E \notin \sigma(L)$ or $E \in \sigma(L(x))$ with $\lambda(E)>0$, there exist two solutions $u^{ \pm} \in R^{\mathbb{Z}}$ of $L u=E u$ which are in $l^{2}( \pm \mathbb{N})$. By Kotani's result [19] and the Lyapunov assumption, this is the case for a set $Y \subset \mathbb{R}$ of full measure that is independent of $x$. The vectors $\Phi^{ \pm}(n)=\left(u^{ \pm}(n+1), u^{ \pm}(n)\right)$ satisfy $A_{E}(n) \Phi^{ \pm}(n)=$ $\Phi^{ \pm}(n+1)$. If $\Phi^{+} \neq \Phi^{-}$, the Titchmarsh-Weyl functions $m_{E}^{ \pm}(n)=u^{ \pm}(n+1) / u^{ \pm}(n)$ are different and real (but they may be infinite). If $\Phi^{+}=\Phi^{-}, E$ is an eigenvalue. The resolvent $(L-E)_{n n}^{-1}=G_{E}(n, n)$ of $L$ satisfies $G_{E}(n, n)=1 /\left(m_{E}^{+}(n)-m_{E}^{-}(n)\right)$ and is the Borel transform of the spectral measure $d k_{e_{n}}$. The absolutely continuous part of $d k_{e_{n}}$ is by Fatou's theorem given by $\operatorname{Im}\left(G_{E+l 0}(n, n)\right)=\operatorname{Im}\left(1 /\left(m_{E+i 0}^{+}(n)-\right.\right.$ $\left.\left.m_{E+10}^{-}(n)\right)\right)$, which is zero for all $E \in Y$ which are not eigenvalues.

Remark. A different proof can be obtained by using a result of Berezanskii (see [25]): if $\mu_{\phi}$ is any spectral measure of any discrete Schrödinger operator $L$ then for $\mu_{\phi}$-almost every $E \in \mathbb{R}$ there exist polynomially bounded solutions of $L u=E u$. If there is an $x \in X$ such that $L(x)$ has absolutely continuous spectrum then it has an absolutely continuous spectral measure $\mu_{\phi}$ supported on a set $A$ of positive Lebesgue measure. For $\mu_{\phi}$-a.e. $E \in A$-which is equivalent to Lebesgue a.e. $E$-the equation $L(x) u=E u$ has polynomially bounded solutions. So $\lambda_{x}(E)=0$ for those $E$. But we assumed that $\lambda_{x}(E)$ does not depend on $x$ and it is strictly positive Lebesgue almost everywhere by Kotani [19]. Thus the assumption that $L(x)$ has absolutely continuous spectrum leads to a contradiction.

Corollary 6.2. If $\left(X_{S}, T\right)$ is a substitution dynamical system, then $L(x)$ has no absolutely continuous spectrum for all $x \in X_{s}$.

Proof. From [15] we know that substitution sequences give operators with Lyapunov exponents independent of $x \in X_{S}$.

Remarks. 1) Another way to exclude absolutely continuous spectrum for all $x$ is to show that the spectrum has Lebesgue measure zero and is independent of $x$. This was done in [4] for circle maps with $\alpha=\beta$. Circle maps give spectrum independent of $x$ for all values of $\alpha$ and $\beta$ [15]. Kotani's theorem [19] does not imply that the spectrum has Lebesgue measure 0 ; a singular spectrum can have positive Lebesgue measure. We do not know whether the Lyapunov exponent is independent of $x$ for sequences generated by circle maps. 
2) One might guess that if $(X, T)$ is a strictly ergodic dynamical system and $V: X \rightarrow \mathbb{R}$ is continuous, then the absolutely continuous spectrum of the operator $(L(x) u)_{n}=u_{n+1}+u_{n-1}+V\left(T^{n} x\right) u_{n}$ is independent of $x \in X$. We have shown that this is the case for dynamical systems $\left(X_{S}, T\right)$ obtained by substitutions if $V\left(T^{n} x\right)=x_{n}$. It is also true for substitution dynamical systems if $V(x)$ only depends on $x_{-l} \cdots x_{l}$, because then the Lyapunov exponent remains independent of $V(x)$ by Proposition 5.2 in [15]. It was already known for circle maps with $\alpha=\beta$ [4] and $V\left(T^{n} x\right)=x_{n}$.

3) Assume that $(X, T)$ is strictly ergodic, that $\lambda_{x}(E)$ exists for all $x \in X$ and is independent of $x$ and that there exists $x \in X$ such that $L(x)$ has no eigenvalues. Then it follows for example from the theorem of de la Vallée Poussin that the spectrum of $L(x)$ is the closure of $\left\{E \mid \lambda_{x}(E)=0\right\}$. We expect (but can't prove) that the spectrum of $L(x)$ is equal to $\left\{E \mid \lambda_{x}(E)=0\right\}$ from which it would follow that it is a Cantor set of zero Lebesgue measure. The spectrum has been shown to be equal to the set $\left\{E \mid \int d \mu(x) \lambda_{x}(E)=0\right\}$, where the integration is with respect to the invariant measure on $X$, for circle maps with $\alpha=\beta$ in [4]. It has been shown that $\operatorname{spec}(L(v))=\left\{E \mid \lambda_{v}(E)=0\right\}$ for a sequence $v$ defined by the Period Doubling substitution in [2] and this result was extended to a class of substitutions including Period Doubling in [5].

\section{The Singular Continuous Spectrum}

Theorem 7.1 (Simon). Let $(X, d)$ be a complete metric space of selfadjoint operators on a Hilbert space $H$ such that the metric $d$ is stronger than the strong resolvent convergence. Then both of the sets $\left\{A \in X \mid \sigma_{p p}=\emptyset\right\},\left\{A \in X \mid \sigma_{a c}=\emptyset\right\}$ are $G_{\delta}$ 's, countable intersections of open sets.

See [24].

Corollary 7.2. Let $X \subset A^{\mathbb{Z}}$ be strictly ergodic. If there is one $x \in X$ such that $L(x)$ has no eigenvalues, then there exists a generic subset in $X$ on which $L$ is purely singular continuous.

Proof. By Kotani [19] there is a set of full measure with no absolutely continuous spectrum. By strict ergodicity, this set is dense and by Theorem 7.1 it is a dense $G_{\delta}$. Since $\operatorname{Orb}(x)$ is dense by minimality, there is dense set on which $L$ has no eigenvalues. Again, this set is a dense $G_{\delta}$ by Theorem 7.1. Since $X$ is a complete metric space, the intersection of two dense $G_{\delta}$ 's is a dense $G_{\delta}$ by the Baire category theorem.

By 5.1, 2.1,3.1 and 4.1 we get:

Corollary 7.3. If $X$ is strictly ergodic and palindromic, then there is a generic $Y \subset$ $X$ such that for $x \in Y$ the operator $L(x)$ has purely singular continuous spectrum.

Corollary 7.4 (Case of substitutions). For substitutions of class $P$, there exists a generic set $Y$ in $X_{S}$ such that for $x \in Y$ the operator $L(x)$ has purely singular continuous spectrum.

Corollary 7.5 (Case of circle maps). For all irrational $\alpha$ and every half-open interval $J$, there exists a generic set $Y$ in $X_{J}$ such that for $x \in Y$ the operator $L(x)$ has purely singular continuous spectrum. 
Remarks. 1) Strictly ergodic palindromic systems other than those defined by circle maps and class $P$ substitutions can be constructed from so-called Toeplitz sequences $[16,21]$.

2) Corollary 7.2 asserts the existence of a generic (hence uncountable) subset in $X$ for which $L$ has purely singular continuous spectrum. Note that for class $P$ substitutions we can actually construct elements of an uncountable set in $X$ for which $L$ has purely singular continuous spectrum, because (i) Corollary 6.2 excludes absolutely continuous spectrum for all $x \in X$ and (ii) the proof of Proposition 2.1 is constructive.

\section{Appendix: The Criterion of Jitomirskaya and Simon}

For the convenience of the reader we repeat the proof of the theorem of Jitomirskaya and Simon [17] for the case of potentials taking finitely many values.

Theorem 8.1 (Jitomirskaya-Simon). Let $x \in A^{\mathbb{Z}}$, where $A \subset \mathbb{R}$ is finite. Consider the operator on $l^{2}(Z)$ given by $(L u)(n)=u(n+1)+u(n-1)+x_{n} u(n)$. Suppose there is a sequence of intervals $I_{i}$ of length $\left|I_{l}\right|$ centered at $m_{l}$ such that $x$ is a palindrome on $I_{i}, 0 \in I_{i}$ and $\left|I_{l}\right| \rightarrow \infty$ as $i \rightarrow \infty$. Then there exists a constant $C$ depending only on $A$ such that $L$ has no eigenvalues if $C^{m_{l}} /\left|I_{l}\right| \rightarrow 0$ as $i \rightarrow \infty$.

Proof. We can assume that $\left|I_{i}\right|$ is either odd or even for all $i$. We prove the theorem for the case that $\left|I_{i}\right|$ is odd and then indicate what changes if it is even. So first let $I_{i}=\left\{m_{i}-l_{l}, m_{l}+l_{i}\right\}$.

Assume $L u=E u$ with $\|u\|=1$. Define $u_{l}(n)=u\left(2 m_{i}-n\right)$, the vector reflected at $m_{l}$. Let $W\left(u, u_{l}\right)(n)=u(n+1) u_{l}(n)-u_{i}(n+1) u(n)$ be the Wronskian of $u$ and $u_{l}$. Let $\Phi(n)=(u(n+1), u(n))$ and $\Phi_{l}(n)=\left(u_{i}(n+1), u_{l}(n)\right)$ and $u_{i}^{ \pm}=u \pm u_{i}$ and $\Phi_{l}^{ \pm}=\Phi \pm \Phi_{l}$.

Step 1. $W(n):=W\left(u, u_{l}\right)(n)$ is constant for $n \in I_{i}$.

Proof. Compute using $u(n+1)=-u(n-1)+\left(E-x_{n}\right) u(n)$ and $u_{i}(n+1)=-u_{i}$ $(n-1)+\left(E-x_{n}\right) u_{i}(n)$,

$$
W(n)-W(n-1)=\left(x_{2 m_{l}-n}-x_{n}\right) u\left(2 m_{i}-n\right) u(n)=0 .
$$

Step 2. $\left|W\left(m_{l}\right)\right| \leqq 2 /\left|I_{l}\right|=2 /\left(2 l_{l}+1\right)$.

Proof. $\sum_{n}|W(n)| \leqq 2$ by Schwarz inequality and $\|u\|=\left\|u_{i}\right\|=1$. Use Step 1 .

Step 3. Either (i) $\left|u_{l}^{+}\left(m_{i}\right)\right| \leqq 2\left|I_{i}\right|^{-1 / 2}$ or (ii) $\left|u_{l}^{-}\left(m_{i}+1\right)\right| \leqq 2\left|I_{l}\right|^{-1 / 2}$.

Proof. Since $u_{i}^{-}\left(m_{l}\right)=0$, one has

$$
u_{i}^{+}\left(m_{l}\right) u_{i}^{-}\left(m_{l}+1\right)=W\left(u_{i}^{-}, u_{i}^{+}\right)\left(m_{i}\right)=2 \cdot W\left(u, u_{l}\right)\left(m_{l}\right)=2 \cdot W\left(m_{l}\right) .
$$

Use Step 2.

Step 4. There exists a constant $C_{1}$ such that $\left\|\Phi_{l}^{ \pm}\left(m_{l}\right)\right\| \leqq C_{1}\left|I_{l}\right|^{-1 / 2}$ holds for all $i$, with either the sign + or - .

Proof. In case (ii), we have $\left\|\Phi_{i}^{-}\left(m_{l}\right)\right\|=\left\|\left(u_{i}^{-}\left(m_{l}+1\right), 0\right)\right\| \leqq 2\left|I_{l}\right|^{-1 / 2}$. Assume now case (i). We have $u_{i}\left(m_{l}\right)=u\left(m_{i}\right)$ and $u_{i}\left(m_{l}-1\right)=u\left(m_{l}+1\right)$, resp. $u_{l}\left(m_{l}+1\right)=u\left(m_{l}-1\right)$ so that $u_{i}^{+}\left(m_{l}-1\right)=u_{i}^{+}\left(m_{l}+1\right)$. Therefore $u_{i}^{+}\left(m_{i}+1\right)=$ $\frac{1}{2}\left(E-x_{m_{l}}\right) u_{i}^{+}\left(m_{l}\right)$ so that $\left\|\Phi^{+}\left(m_{l}\right)\right\| \leqq C_{1}^{\prime}\left|u^{+}\left(m_{l}\right)\right| \leqq C_{1} \cdot\left|I_{l}\right|^{-1 / 2}$. 
Step 5. For some $C>0,\left\|\Phi_{l}^{ \pm}(0)\right\| \leqq C^{m_{i}} C_{1} \cdot\left|2 l_{1}+1\right|^{-1 / 2}$, for either sign + or sign -.

Proof. There exists $C$ such that $\|A(n)\| \leqq C$ for all transfer matrices $A(n)$, for all $E$ in the spectrum of $L$. Denote by $A_{i}$ the transfer matrix that maps vectors at $m_{i}$ to vectors at 0 . In particular, because $x$ is exactly symmetric on $I_{i}$,

$$
A_{i} \Phi\left(m_{i}\right)=\Phi(0), \quad A_{l} \Phi_{i}\left(m_{i}\right)=\Phi_{i}(0) .
$$

Then also

$$
\Phi_{i}^{ \pm}(0)=A_{l} \Phi_{i}^{ \pm}\left(m_{i}\right)
$$

so that

$$
\left\|\Phi_{l}^{ \pm}(0)\right\| \leqq C^{m_{l}} C_{1} \cdot\left|2 l_{1}+1\right|^{-1 / 2} .
$$

Step 6. Proof of the theorem.

By Step 5,

$$
\left|\|\Phi(0)\|-\left\|\Phi\left(2 m_{i}\right)\right\|\right| \leqq\left\|\Phi^{ \pm}(0)\right\| \rightarrow 0
$$

for suitable choices of + and - as $i \rightarrow \infty$. Since $\left\|\Phi\left(m_{i}\right)\right\| \rightarrow 0$ as $m_{l} \rightarrow \infty$ because $u \in l^{2}$, it follows that $\Phi(0)=0$. But then $u=0$, contradicting $\|u\|=1$. If $I_{i}$ is even for all $i$ we can put $I_{l}:=\left\{m_{i}-l_{i}, \ldots, m_{l}, m_{i}+1, \ldots, m_{l}+l_{i}+1\right\}$. Define $u_{i}(n)=$ $u\left(2 m_{l}-n+1\right)$, at $m_{i}+1 / 2$ reflected vector. We have $x_{2 m_{l}-n+1}=x_{n}$ on $I_{i}$.

Step 1 and Step 2 do not change.

In Step 3, the claim stays the same. Note that $u_{i}^{-}\left(m_{i}\right)=0$ is no longer true in general. However, the claim follows from

$$
W\left(u_{l}^{-}, u_{i}^{+}\right)=2 u_{i}^{+}\left(m_{l}\right) u_{i}^{-}\left(m_{i}+1\right)
$$

(even with a factor 2 deleted), which is a consequence of $u_{i}^{+}\left(m_{l}\right) u_{i}^{-}\left(m_{i}+1\right)=$ $-u_{i}^{+}\left(m_{i}+1\right) u_{i}^{-}\left(m_{l}\right)$.

Step 4. In case (ii) we take

$$
\left\|\Phi_{l}^{+}\left(m_{i}\right)\right\|=\left\|\left(u_{i}^{+}\left(m_{i}+1\right), u_{i}^{+}\left(m_{i}\right)\right)\right\| \leqq \sqrt{2} \cdot\left|u_{i}^{+}\left(m_{i}\right)\right|,
$$

since $u_{i}^{+}\left(m_{i}\right)=u_{i}^{+}\left(m_{i}+1\right)$. Case (i) with a different $C_{1}$ follows from $u_{i}^{-}\left(m_{i}\right)=$ $-u_{\imath}^{-}\left(m_{i}+1\right)$

Step 5. Define $A_{l}$ by $A_{l} \Phi\left(m_{i}\right)=\Phi(0)$. Because $x$ is symmetric on $I_{l}$, we have

$$
A_{l} A\left(m_{l}\right) \Phi\left(m_{l}\right)=A_{i} \Phi\left(m_{i}+1\right)=\Phi\left(2 m_{l}+1\right)=\Phi_{l}(0) .
$$

Together

$$
\left\|\Phi_{i}^{ \pm}(0)\right\|=\left\|\Phi(0) \pm \Phi_{i}(0)\right\| \leqq 2 C^{m_{l}+1}\left\|\Phi^{ \pm}\left(m_{i}\right)\right\|
$$

for either the sign + or the sign - .

Step 6 is the same (replace $\Phi\left(2 m_{l}\right)$ by $\left.\Phi\left(2 m_{1}+1\right)\right)$.

Note added in proof. Y. Last and B. Simon have now a proof of a result, which implies the guess done in Remark 2) Section 6 of this paper. 


\section{References}

1. Bellissard, J.: Spectral properties of Schrödinger's operator with Thue-Morse potential. In: Luck, J.M., Moussa, P., Waldschmidt, M. (eds.) Number Theory and Physics, Springer Proceedings in Physics, Vol. 47, Berlin, Heidelberg, New York: Springer 1990, pp 140-150

2. Bellissard, J., Bovier, A., Ghez, J.-M.: Spectral properties of a tight binding Hamiltonian with period doubling potential. Commun. Math. Phys. 135, 379-399 (1991)

3. Bellissard, J., Bovier, A., Ghez, J.-M.: Gap labelling theorems for one dimensional discrete Schrödinger operators. Rev. Math. Phys. 4, 1-37 (1992)

4. Bellissard, J., Iochum, B., Scoppola, E., Testard, D.: Spectral properties of one dimensional quasi-crystals. Commun. Math. Phys. 125, 527-543 (1989)

5. Bovier, A., Ghez, J.-M.: Spectral properties of one-dimensional Schrödinger operators with potentials generated by substitutions. Commun. Math. Phys. 158, 45-66 (1993)

6. Bovier, A., Ghez, J.-M.: Erratum to [5]. Commun. Math. Phys.

7. Carmona, R., Klein, A., Martinelli, F.: Anderson localization for Bernoulli and other singular potentials. Commun. Math. Phys. 108, 41-66 (1987)

8. Casdagli, M.: Symbolic dynamics for the renormalization map of a quasiperiodic Schrödinger equation. Commun. Math. Phys. 107, 295-318 (1986)

9. Dekking, F.M.: The spectrum of dynamical systems arising from substitutions of constant length. Z. Wahr. Verw. Geb. 41, 221-239 (1978)

10. Delyon, F., Petritis, D.: Absence of localization in a class of Schrödinger operators with quasiperiodic potential. Commun. Math. Phys. 103, 441-444 (1986)

11. Delyon, F., Peyrière, J.: Recurrence of the eigenstates of a Schrödinger operator with automatic potential. J. Stat. Phys. 64, 363-368 (1991)

12. Dulea, M., Johansson, M., Riklund, R.: Localization of electrons and electromagnetic waves in a deterministic aperiodic system. Phys. Rev. B45, 105-114 (1992)

13. Dulea, M., Johansson, M., Riklund R.: Unusual scaling of the spectrum in a deterministic aperiodic tight-binding model. Phys. Rev. B47, 8547-8551 (1993)

14. Hof, A.: Quasicrystals, aperiodicity and lattice systems. Proefschrift, Rijksuniversiteit Groningen, 1992

15. Hof, A.: Some remarks on discrete aperiodic Schrödinger operators. J. Stat. Phys. 72, 13531374 (1993)

16. Jacobs, K., Keane, M.: 0-1-sequences of Toeplitz type. Z. Wahr. Verw. Geb. 13, 123-131 (1969)

17. Jitomirskaya, S., Simon, B.: Operators with singular continuous spectrum: III. Almost periodic Schrödinger operators. Commun. Math. Phys. 165, 201-205 (1994)

18. Kohmoto, M., Kadanoff, L.P., Tang, C.: Localization problem in one dimension: Mapping and escape. Phys. Rev. Lett. 50, 1870-1872 (1983)

19. Kotani, S.: Jacobi matrices with random potential taking finitely many values. Rev. Math. Phys. 1, 129-133 (1989)

20. Luck, J.M.: Cantor spectra and scaling of gap widths in deterministic aperiodic systems. Phys. Rev. B39, 5834-5849 (1989)

21. Neveu, J.: Sur les suites de Toeplitz. Z. Wahr. Verw. Geb. 13, 132-134 (1969)

22. Ostlund, S., Pandt, R., Rand, D., Schellnhuber, H.J., Siggia, E.D.: One-dimensional Schrödinger equation with an almost periodic potential. Phys. Rev. Lett. 50, 1873-1876 (1983)

23. Queffélec, M.: Substitution Dynamical Systems-Spectral Analysis, volume 1294, Lecture Notes in Mathematics. Berlin, Heidelberg, New York: Springer, 1987

24. Simon, B.: Operators with singular continuous spectrum: I. General operators. To appear in Ann. Math.

25. Simon, B.: Schrödinger semigroups. Bull. Amer. Math. Soc. 7, 447-526 (1982)

26. Sütö, A.: The spectrum of a quasiperiodic Schrödinger operator: Commun. Math. Phys. 111, 409-415 (1987)

27. Sütö, A.: Singular continuous spectrum on a Cantor set of zero Lebesgue measure for the Fibonacci Hamiltonian. J. Stat. Phys. 56, 525-531 (1989) 
